\title{
Brief Report: Sedatives for Mothers of Stillborn Infants: Views from a National Survey of Obstetricians
}

\author{
Katherine J. Gold, M.D., M.S.W., M.S., ${ }^{1,2}$ Thomas L. Schwenk, M.D., ${ }^{1}$ and Timothy R.B. Johnson, M.D. ${ }^{2}$
}

\begin{abstract}
Although no guidelines support the use of benzodiazepines in bereavement care, clinicians continue to use this medication for surviving family members. An anonymous survey about perinatal death was sent to 1500 United States obstetricians. One question assessed beliefs about prescribing sedatives to bereaved mothers. Almost half $(49 \%)$ of 804 obstetricians endorsed the use of sedatives, such as benzodiazepines, for acute bereavement. Grieving families may experience mental and physical anguish after a death; however, benzodiazepines are not part of recommended care for bereavement. The surprisingly high support from physicians for the use of sedatives in the case of maternal grief raises important questions about how physicians can best support bereaved mothers and what effect benzodiazepines may have in the grieving process.
\end{abstract}

\section{Introduction}

A NECDOTAL REPORTS SUGGEST physicians sometimes prescribe benzodiazepines as treatment for acutely bereaved patients. ${ }^{1-3}$ We report on a large national survey of obstetricians; the broader study measured physician experiences with perinatal death, adequacy of their training in this area, and coping patterns. The survey also assessed provider attitudes about prescribing benzodiazepines after perinatal death, generally defined as fetal death in the second half of pregnancy or in the first 28 days after birth.

\section{Materials and Methods}

We conducted an anonymous mail survey of 1500 United States obstetricians from June through September 2007. Names were randomly selected from the American Medical Association (AMA) Masterfile and limited to physicians holding an active medical license. We replaced physicians if mailings were returned undeliverable. Respondents were sent up to three copies of the survey and a stamped return envelope, one reminder postcard, and a $\$ 2$ cash incentive with the initial mailing. The study was approved by the institutional review board at the University of Michigan.

The survey included 51 questions about physician beliefs and experiences regarding coping with the death of a stillborn infant or infant death. One question stated: "After a stillbirth, I believe it can be helpful to offer sedatives (such as benzodiazepines) to a grieving mother to help her cope with her loss." Results from this single question are pre- sented in this brief communication to allow fuller discussion of an important issue in bereavement care.

\section{Results}

Of 1500 physicians, 19 envelopes were undeliverable and names were replaced; 15 envelopes came back undeliverable after the close of the survey period and were not replaced. Of 1485 completed mailings, 33 physicians returned a form declining to participate, 2 returned incomplete surveys, and 804 completed the survey, for a usable response rate of $54 \%$. Respondents were $51 \%$ female; $74.5 \%$ self-identified as white/Caucasian, $11.3 \%$ as Asian/Pacific Islander, $6.6 \%$ as black/African American, and 7.6\% as other race/ethnicity or unknown. Median age was 46 , with an average of 14 years experience after residency; $86 \%$ were attending physicians, and $14 \%$ were residents or fellows.

For the question about whether sedatives can be helpful to a grieving mother, $8 \%$ marked strongly disagree, $42 \%$ disagree, $42 \%$ agree, and 6.5\% strongly agree (1.5\% missing). Thirteen physicians left the question blank. Female physicians were less likely to endorse the use of sedatives (OR 0.53, CI 0.40-0.71, $p<0.001)$. Female gender remained significant (OR 0.65, CI 0.47-0.90, $p=0.009$ ) even after controlling for age, race, position (resident, fellow, attending), and whether the physician reported adequate preparation to help patients with grief and bereavement issues after perinatal death. Older physician age also predicted support for sedatives in both bivariable and multivariable models. Age and years experience were collinear, so substitution did not

${ }^{1}$ Department of Family Medicine, and ${ }^{2}$ Department of Obstetrics \& Gynecology, University of Michigan, Ann Arbor, Michigan. 
change the results. Belief that sedatives could be helpful was not related to race, position (attending vs. trainee), being a parent, personal/family experience with perinatal loss, a belief that one's training was inadequate, greater death anxiety, or personal use of substances, including prescription medications, to cope after a death.

\section{Discussion}

Half of the obstetricians in our survey indicated prescribing sedative medications, such as benzodiazepines, could be useful to grieving parents after the death of a baby. A few small studies have noted similar findings. Cook et al. ${ }^{1}$ recently expressed concern that 18 of 33 physicians in their study (of primary care or geriatric physicians) spontaneously reported prescribing benzodiazepines for bereavement. Of 112 family medicine physicians in a separate study, 55\% reported they would prescribe psychotropic medications to newly bereaved parents for a short period. ${ }^{2}$ Fifteen of 37 parents in a 1994 survey reported they were prescribed benzodiazepines after a stillbirth or infant death. ${ }^{3}$

Although some patients are unaffected by short-term use of benzodiazepines, a subset-perhaps as many as $10 \%-15 \%$ - will continue using these medications on a longterm basis. ${ }^{4}$ Psychiatric comorbidity, less education, loneliness, older age, use of a benzodiazepine with a short halflife, and repeat prescriptions all appear to be associated with long-term use, although there is limited research in this area. $^{4,5}$ Studies show mixed results on whether initial dosage affects risk of long-term use. ${ }^{4}$ Benzodiazepines are commonly used for difficulty sleeping; however, there are alternative treatments for insomnia, and a large meta-analysis has shown significant potential for side effects, including daytime sedation, dizziness, and lightheadedness. ${ }^{6}$

The only randomized, controlled trial to date, a small study to evaluate the use of benzodiazepines after bereavement, demonstrated no benefit for grief or sleep. ${ }^{7}$ Although a different dose could theoretically lead to a different outcome, even at low doses, the study results suggested that individuals treated with benzodiazepines had more difficulty resolving sleep problems in the 2 months after bereavement.

In a survey of bereaved adults, half of respondents agreed or strongly agreed that medication could help with sleep after bereavement, but two thirds thought it would also delay coming to terms with the loss. ${ }^{7}$ Indeed, the British National Formulary recommends against the use of benzodiazepines for bereavement because of concern that these medications could inhibit normal grieving. ${ }^{7}$ Although there are likely differences in benzodiazepine use internationally, a survey of psychiatric experts from 44 countries found that $48 \%$ agreed benzodiazepines were indicated as basic treatment for anxiety following bereavement, suggesting these views may be widespread. ${ }^{8}$

Like all cross-sectional surveys, ours was limited by potential nonresponse bias. Nonresponders were not surveyed, but respondent age and race were similar to national demographic data for obstetricians. ${ }^{9,10}$ We had a low response rate for a national survey, although it was higher than the typical $52 \%$ rate of large, national physician surveys. ${ }^{11}$ Finally, the survey measured opinion and may not reflect actual practice, response to patient requests, beliefs about ideal length of drug use, or perception of low patient risk for drug side effects and dependence. These questions should be evaluated in future studies of bereavement care.

This large, nationally representative study found surprisingly widespread obstetrician support for sedative use in cases of acute bereavement. Although grieving families may experience both mental and physical anguish after a death, benzodiazepines are not part of recommended care for families. The addictive nature of these medications poses a risk to some patients and could potentially interfere with coping and subsequent decision making. Given the lack of evidence supporting use in bereavement and the significant potential for adverse effects, we respectfully encourage providers to be aware of this practice and consider other interventions to support grieving patients and families.

\section{Acknowledgments}

The study was funded by the Robert Wood Johnson Foundation's Clinical Scholars Program and the University of Michigan Department of Obstetrics \& Gynecology. Neither organization had any direct role in the design and conduct of the study; collection, management, analysis, or interpretation of the data; or preparation, review, or approval of the manuscript. As the principal investigator, K.J.G. had full access to all the data in the study and takes responsibility for the integrity of the data and the accuracy of the data analysis. All authors had full access to the data.

\section{Disclosure Statement}

No competing financial interests exist.

\section{References}

1. Cook JM, Biyanova T, Marshall R. Medicating grief with benzodiazepines: Physician and patient perspectives. Arch Intern Med 2007;167:2006-2007.

2. Lemkau JP, Mann B, Little D, Whitecar P, Hershberger P, Schumm JA. A questionnaire survey of family practice physicians' perceptions of bereavement care. Arch Fam Med 2000;9:822-829.

3. Harper MB, Wisian NB. Care of bereaved parents: A study of patient satisfaction. I Reprod Med 1994;39:80-86.

4. Van Hultan R, Teeuw KB, Bakker A, Leufkens HG. Initial 3month usage characteristics predict long-term use of benzodiazepines: An 8-year follow-up. Eur J Clin Pharmacol 2003;58:689-694.

5. Zandstra SM, van Rijswijk E, Rijnders CATh, et al. Longterm benzodiazepine users in family practice: Differences from short-term users in mental health, coping behaviour and psychological characteristics. Fam Pract 2004;21: 266-269.

6. Holbrook AM, Crowther R, Lotter A, Cheng C, King D. Meta-analysis of benzodiazepine use in the treatment of insomnia. Can Med Assoc J 2000;162:225-233.

7. Warner J, Metcalfe C, King M. Evaluating the use of benzodiazepines following recent bereavement. $\underline{\mathrm{Br} J}$ Psychiatry 2001;178:36-41.

8. Balter MB, Ban TA, Uhlenhuth EH. International study of expert judgment on therapeutic use of benzodiazepines and other psychotherapeutic medications: I. Current concerns. Hum Psychopharmacol 1993;8:253-261. 
9. American Medical Association. Physician characteristics and distribution in the United States, 2007 ed. Chicago, IL: American Medical Association, 2007.

10. Frank E, Rock J, Sara D. Characteristics of female obstetrician-gynecologists in the United States. Obstet Gynecol. 1999;94:659-665.

11. Cummings SM, Savitz LA, Konrad TR. Reported response rates to mailed physician questionnaires. Health Serv Res 2001;35:1347-1355.
Address reprint requests to: Katherine J. Gold, M.D., M.S.W., M.S. Department of Family Medicine Department of Obstetrics \& Gynecology 1018 Fuller Street University of Michigan Ann Arbor, MI 48104-1213 E-mail: ktgold@umich.edu 